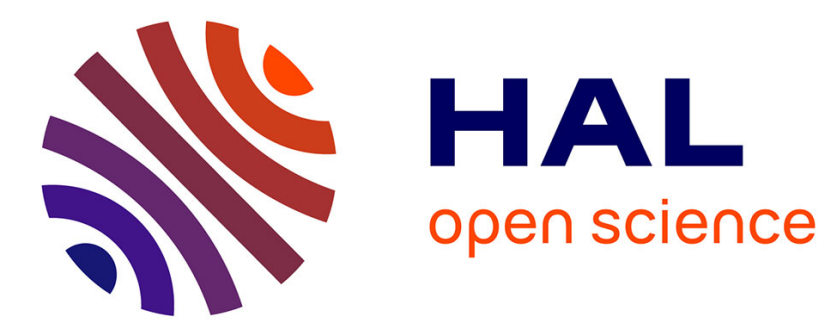

\title{
Examples of automatic asymptotic expansions Bruno Salvy
}

\section{To cite this version:}

Bruno Salvy. Examples of automatic asymptotic expansions. RT-0114, INRIA. 1989, pp.16. inria00070052

\section{HAL Id: inria-00070052 \\ https://hal.inria.fr/inria-00070052}

Submitted on 19 May 2006

HAL is a multi-disciplinary open access archive for the deposit and dissemination of scientific research documents, whether they are published or not. The documents may come from teaching and research institutions in France or abroad, or from public or private research centers.
L'archive ouverte pluridisciplinaire HAL, est destinée au dépôt et à la diffusion de documents scientifiques de niveau recherche, publiés ou non, émanant des établissements d'enseignement et de recherche français ou étrangers, des laboratoires publics ou privés. 


\section{Rapports Techniques}

$\mathrm{N} \bullet 114$

UNITÉ DE RECHERCHE INRIA-ROCQUENCOURT

Institut National de Recherche en Informatique et en Automatique

Domaine de Voluceau Rocquencourt B.P. 105

78153 Le Chesnay Cedex France Tél.:(1)3963 5511

EXAMPLES OF AUTOMATIC ASYMPTOTIC EXPANSIONS

Bruno SALVY

Décembre 1989 


\title{
Examples of Automatic Asymptotic Expansions
}

\author{
Bruno Salvy
}

\begin{abstract}
We describe the current state of a Maple library, gdev, designed to perform asymptotic expansions for a large class of expressions. Many examples are provided, along with a short sketch of the underlying principles. At the time when this report is written, a striking feature of these examples is that none of them can be computed directly with any of today's most widespread symbolic computation systems (Macsyma $^{\dagger}$, Mathematica $^{\ddagger}$, Maple $^{\S}$ or Scratchpad II ${ }^{\Uparrow}$ ).
\end{abstract}

\section{Exemples de développements asymptotiques automatiques}

\begin{abstract}
Résumé
Nous décrivons l'état actuel d'une bibliothèque Maple - gdev—conçue pour calculer automatiquement des développements asymptotiques, et ce pour une classe d'expressions étendue. De nombreux exemples sont présentés, ainsi qu'une brève description des principes sous-jacents. A l'heure actuelle, aucun de ces exemples ne peut être obtenu de façon directe sur les systèmes de calcul formel les plus développés (Macsyma ${ }^{\dagger}$, Mathematica ${ }^{\ddagger}$, Maple ${ }^{\S}$ ou Scratchpad II ${ }^{\mathbb{T}}$ ).
\end{abstract}

\footnotetext{
†Sun Release 309.6

$\ddagger$ Version 1.2 , beta test

$\S$ Version 4.3

IV Version $4 \mathrm{~J}$
} 


\title{
Examples of Automatic Asymptotic Expansions
}

\author{
Bruno Salvy \\ LIX, Ecole Polytechnique \\ 91128 Palaiseau Cedex (France) \\ and \\ INRIA, Rocquencourt \\ 78150 Le Chesnay (France)
}

\begin{abstract}
We describe the current state of a Maple library, gdev, designed to perform asymptotic expansions for a large class of expressions. Many examples are provided, along with a short sketch of the underlying principles. At the time when this report is written, a striking feature of these examples is that none of them can be computed directly with any of today's most widespread symbolic computation systems (Macsyma $^{\dagger}$, Mathematica $^{\ddagger}$, Maple $^{\S}$ or Scratchpad II ${ }^{\Uparrow}$ ).
\end{abstract}

\section{Introduction}

Current symbolic computation systems generally lack facilities for manipulating asymptotic expansion computations of a form more complex than the first terms of a Taylor series or a Puiseux expansion (involving fractional powers). We introduce a set of programs whose aim is to contribute to fill this gap. The emphasis here is on examples displaying the variety of difficulties a general purpose program must be prepared to encounter. With each of these examples is given a glimpse of the built-in choices which make these procedures work where others won't.

All these examples have been attempted using the built-in asymptotic capabilities of Maple, Mathematica, Macsyma and Scratchpad. In all the cases (although Maple seems to perform better on simpler examples) none of these systems could solve the problem directly, their answers ranging from non-evaluated expressions or error messages to segmentation faults or (apparently) infinite computations.

In the first part of this report, we give an overview of automatic asymptotic expansions of functions given in an explicit form. Once this is obtained, it becomes feasible to implement methods that deal with non explicit expressions. Two such applications are presented in part 2 and 3 . The first one is specialized in discrete problems for which a generating function is available and the second one deals with a large class of expressions given as integrals. In these two last parts, not many details are given and the reader is supposed to know or to accept the principles of the methods which are implemented.

\section{Foreword}

In all the following examples, the toplevel procedure is gdev, which stands for "generalized development". Its synopsis is:

$\operatorname{gdev}(\operatorname{expr}, \operatorname{var}=1$ impoint,$\langle$ nbterms $>,\langle\operatorname{dir}\rangle)$

\footnotetext{
${ }^{\dagger}$ Sun Release 309.6

$\ddagger$ Version 1.2 , beta test

$\S$ Version 4.3

IV Version 4J
} 
where

- expr is the expression to be studied,

- var=limpoint is an equation indicating the variable with respect to which the expansion will be computed and the point this variable is approaching, this point being any complex point or plus or minus infinity,

- nbterms is an optional parameter specifying the number of terms required in the expansion (the default is 1 ),

- dir is an optional parameter specifying the direction taken by the variable to approach its limit. This may be one of straight or inverse. The former means that the variable tends to its limiting point following a straight line starting from 0 and the latter means that it follows a straight line coming from infinity. At the origin, 'straight' means from the right on the real axis and 'inverse' means from the left. At plus or minus infinity, the variable always comes from 0 . The default is 'straight'.

Another procedure glimit is built on top of gdev and computes only the limit of the expression. The synopsis is the same, except that no number of terms is required.

\section{Expansions of expressions given in a closed form}

Usually systems perform well on Puiseux scales and poorly on more general scales. It is then necessary to define clearly what is meant by 'asymptotic scale' and 'asymptotic expansion'.

Definition 1 Let $\mathcal{P}$ be a point set and $\mathcal{L}$ a limiting point of $\mathcal{P}$. Let $\mathcal{S}_{\mathcal{P}}$ be a set of functions defined on $\mathcal{P}$, with values in the complex plane. $\mathcal{S}_{\mathcal{P}}$ is said to be an asymptotic scale at $\mathcal{L}$ when for any couple $\left(\varphi_{1}, \varphi_{2}\right)$ of functions in $\mathcal{S}_{\mathcal{P}}$, the limit of $\varphi_{1}(x) / \varphi_{2}(x)$ when $x$ tends to $\mathcal{L}$ in $\mathcal{P}$ is defined and is one of $0,+\infty$.

Definition 2 Let $\mathcal{S}_{\mathcal{P}}$ be an asymptotic scale at $\mathcal{L}$ and $f$ a complex valued function defined on $\mathcal{P}$. Then $f$ is said to admit an asymptotic expansion of Poincaré type at $\mathcal{L}$ when there exist an $n$-tuple $\left(a_{1}, \ldots, a_{n}\right)$ of complex numbers and an n-tuple $\left(\varphi_{1}, \ldots, \varphi_{n}\right)$ of elements of $\mathcal{S}_{\mathcal{P}}$ such that

$$
\forall i \in\{1, \ldots n\}, \forall \varphi \in \mathcal{S}_{\mathcal{P}} \quad \varphi_{i}=o(\varphi) \Longrightarrow f-\sum_{j=1}^{i} a_{j} \varphi_{j}=o(\varphi)
$$

Usually, scales at a finite point $a$ with which systems are at ease include $\left\{(x-a)^{n}, n \in \mathbf{N}\right\}$ (Taylor scale), $\left\{(x-a)^{p}, p \in \mathbf{Z}\right\}$ (Laurent scale) or $\left\{(x-a)^{r}, r \in \mathbf{Q}\right\}$ (Puiseux scale) and the analogous of these scales at plus or minus infinity.

Beyond these scales, systems are usually at a loss, being unable to determine automatically in which scale the computation must be performed. The following examples show some typical instances of what basic cases our library is able to handle, that were unobtainable previously.

\section{I.1 Examples at a finite distance on the real axis}

\section{a. A logarithmic scale}

EXAMPle 1:

$>\operatorname{gdev}(\ln (\cot (-x / \ln (x))), x=0,3)$; 


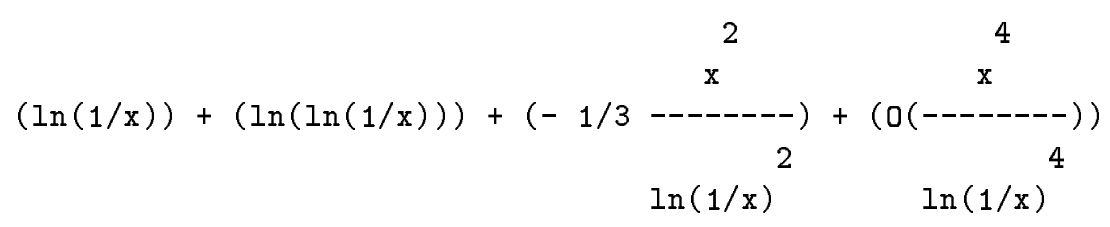

Here, the program automatically determines that the expansion's natural scale is

$$
\{-\ln x, \ln (-\ln x)\} \cup\left\{\left(\frac{x}{-\ln x}\right)^{k}\right\}_{k \in \mathbf{N}} .
$$

\section{b. An exponential scale}

EXAMPLE 2:

$>\operatorname{gdev}(\exp (\tan (\mathrm{x}))-1, \mathrm{x}=\mathrm{Pi} / 2,2)$;

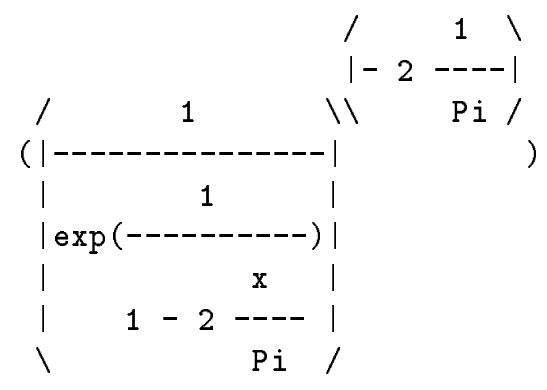

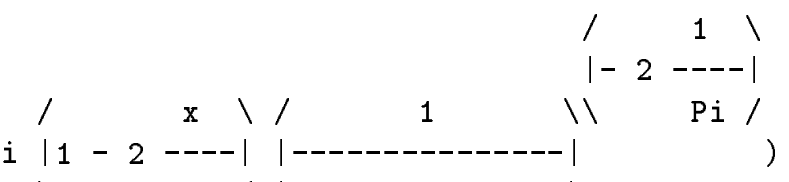

$$
\begin{aligned}
& \begin{array}{rlrl}
+(-1 / 6 \mathrm{Pi} \mid 1-2 & ----\mid & \mid-1 \\
& & \mathrm{Pi} / & \mid \\
& & & \mid \exp (---------)
\end{array} \\
& \left|\begin{array}{c}
x \\
1-2--
\end{array}\right| \\
& \text { । } \mathrm{Pi} /
\end{aligned}
$$

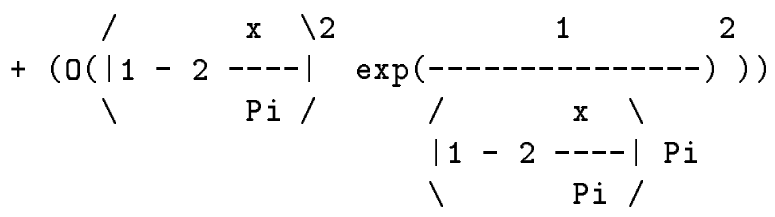

In this example, there is an essential singularity at $\pi / 2$ and the natural scale for this expansion was

$$
\left\{\left(1-\frac{x}{\pi / 2}\right)^{k} e^{\frac{2}{\pi-2 x}}\right\}_{k \in \mathbf{N}}
$$




\section{c. An extended definition of asymptotic expansions}

Many extensions of the definition of asymptotic expansions given above appear in the literature (see e.g. $[5,15])$ and we choose an intermediate one which allows more general expansions without sacrificing implementability: In our program, the coefficients $a_{i}$ are allowed to be functions of bounded variation. The following example shows a use of this extended definition:

EXAMPLE 3:

$>\operatorname{gdev}\left(x^{\wedge} 2 * \sin (1 / x) / \sin (x), x=0,2\right)$;

$$
(\sin (1 / x) x)+\left(1 / 6 \sin (1 / x) x^{3}\right)+\left(0\left(x^{5}\right)\right)
$$

\section{d. A careful look at orders of expansion}

One must be very cautious concerning the management of the orders of the expansions of the subexpressions, this is critical in examples with numerous cancellations. In Maple, the given order is the order taken in the intermediate computations, to get the following result, it is necessary to specify an order of 44 ! In Mathematica, the program does not seem to terminate.

EXAMPLE 4:

$>\operatorname{gdev}\left(\left(\tan \left(\sin \left(x^{\wedge} 4\right)\right)-\sin \left(\tan \left(x^{\wedge} 4\right)\right)\right) / x^{\wedge} 28, x=0,2\right)$;

$$
(1 / 30)+\left(\begin{array}{c}
298 \\
756
\end{array}\right.
$$

This example needs another comment. Some systems have chosen to compute expansions with an absolute order. However, as soon as we allow expansions in more general scales, there is no other choice than to define the order as the number of terms in the expansion.

\section{I.2 At infinity}

There is no real difference between expansions at a finite point and expansions at infinity. The following example involves an intermediate expansion in the scale $\left\{\exp (-n x) / x^{n}\right\}$ which does not appear in the result:

EXample 5:

$>f:=\ln (x)^{-} 2-\ln (x) * \ln (x+\exp (-x))+\arctan (\ln (x))$;

$$
f:=\ln (x)^{2}-\ln (x) \ln (x+\exp (-x))+\arctan (\ln (x))
$$

$>\operatorname{gdev}(f, x=$ inf inity, 2$)$;

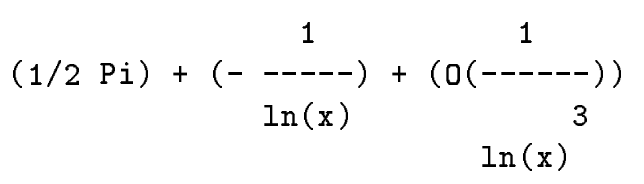


Example 6: Euler's $\Gamma$ function is defined as

$$
\Gamma(x)=\int_{0}^{\infty} e^{-t} t^{x-1} d t
$$

and is known in Maple as GAMMA. Asymptotic expansions of expressions involving it often necessitate difficult intermediate scales, even though they may not appear in the result. In the following example, the final result necessitates an "extended" expansion:

$>f:=\operatorname{GAMMA}(x+\sin (x)) / \operatorname{GAMMA}(x) * x^{\wedge}(-\sin (x))$;

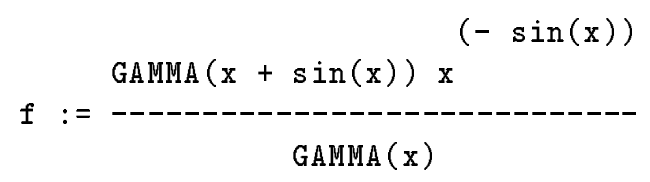

$>\operatorname{gdev}(f, x=$ infinity, 2$)$;

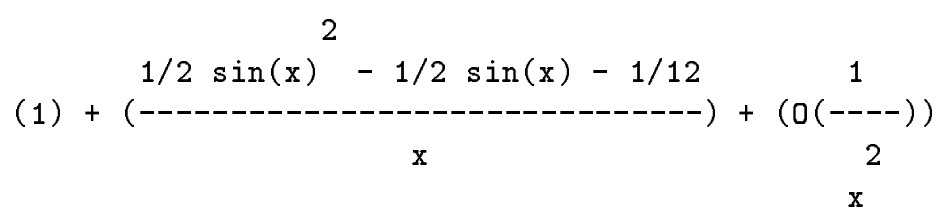

Example 7: The $W$ function is defined in Maple by

$$
W(x) \exp (W(x))=x .
$$

The limit and the scale of the following expansion are not totally obvious by hand:

$>\operatorname{gdev}\left(x^{\wedge}(1 / w(x)), x=\right.$ infinity, 2$)$;

$$
(\exp (1))+\left(\begin{array}{cc}
\exp (1) \ln (\ln (x)) & \ln (\ln (x))^{2} \\
\hline \ln (x) & 2^{2}
\end{array}\right.
$$

EXAmple 8: The following function is taken from Hardy [8] who uses it to describe the difficulty of finding a scale:

$>\mathrm{f}:=\operatorname{sqrt}(\mathrm{x}) *(\ln (\mathrm{x}))^{\wedge} 2 * \exp \left(\operatorname{sqrt}(\ln (\mathrm{x})) *(\ln (\ln (\mathrm{x})))^{\wedge} 2 * \exp (\operatorname{sqrt}(\ln (\ln (\mathrm{x}))) *\right.$

$>(\ln (\ln (\ln (x))))-3))$ :

which reads

$$
f=\sqrt{x} \ln ^{2} x \exp \left\{\sqrt{\ln x}(\ln \ln x)^{2} \exp \left[\sqrt{\ln \ln x}(\ln \ln \ln x)^{3}\right]\right\} .
$$

Then the following limit is difficult to obtain:

$>\operatorname{glimit}(f / \exp (x), x=\operatorname{infinity})$; 
What happens is that the only simple scale in which this function can be expanded is a scale containing the function itself:

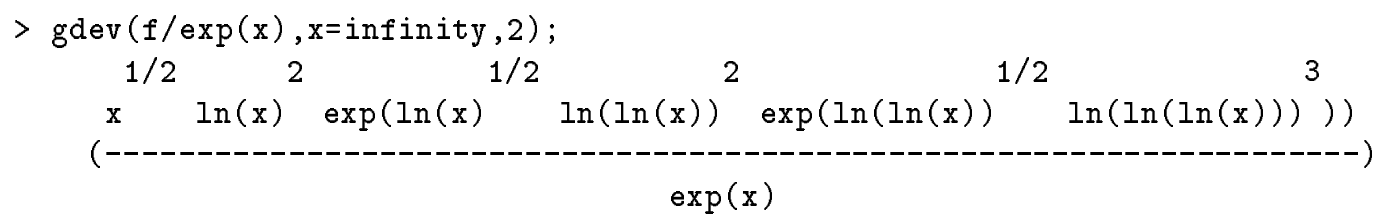

Of course special functions can be handled by the same tools and sometimes they even need our generalized definition of an expansion:

EXAMPLE 9:

$>\operatorname{gdev}(\operatorname{BesselJ}(2, \ln (x)), x=\operatorname{infinity}, 2)$;

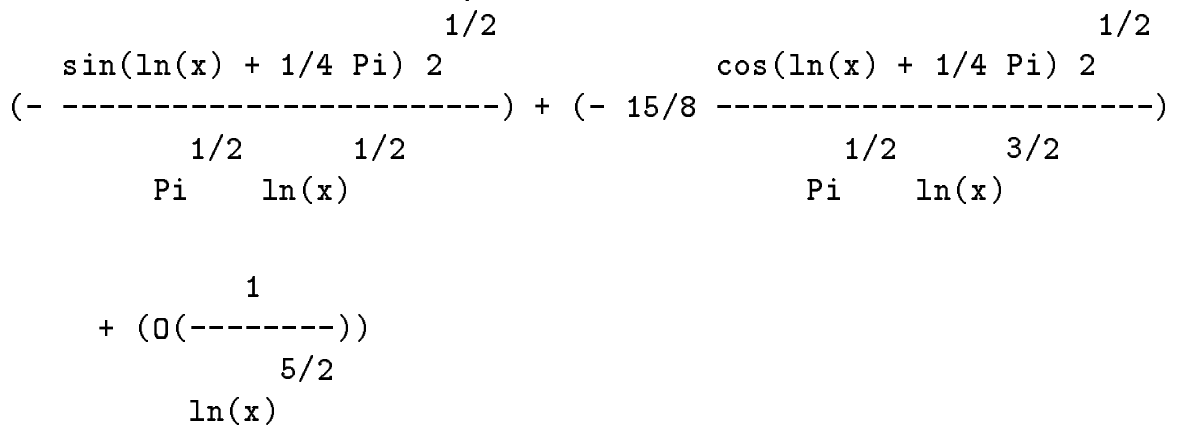

\section{I.3 With parameters}

Parameters only make worse the comparison problem for constants. Since, by a theorem of Richardson [13], this problem is undecidable in general, tools have to be developed that handle more or less extensive classes of expressions.

EXAMPLE 10:

$>$ bin:=binomial $(p+n-w-1, n-w-1) /$ binomial $(p+n-1, n)$; binomial $(\mathrm{p}+\mathrm{n}-\mathrm{w}-1, \mathrm{n}-\mathrm{w}-1)$

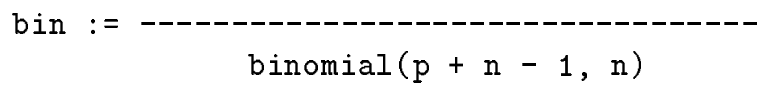

The reader will notice that the coefficients in the above expansion could be simplified further. Our library deliberately adopts a conservative attitude by leaving simplification to the system. The user can use the tools provided by Maple to get the result in his preferred form.

\section{I.4 When the variable is an integer}

Expansions with an integer variable may be defined when the expression is not valid for the continuous variable. An automatic handling of equivalence classes is then often necessary: 
EXAMPLE 11:

$>\mathrm{t}:=1+\cos (\mathrm{n} * \mathrm{Pi} / 2)+1 / \mathrm{n}:$

$>\operatorname{gdev}(\ln (t)$, infinity);

When $\mathrm{n}$ modulo $2=1$ the result is:

$(1 / \mathrm{n})+\left(\begin{array}{c}1 \\ 0(----))\end{array}\right.$

2

n

When $\mathrm{n}$ modulo $4=0$ the result is:

$(\ln (2))+(0(1 / n))$

When $\mathrm{n}$ modulo $4=2$ the result is:

$(-\ln (n))$

The splitting into equivalence classes arises when a constant (i.e. an expression independent of the main variable) is possibly zero. We modified the standard solver of Maple so that in a number of cases, it can return an "infinite" list, and then a few procedures manage these infinite lists and perform the necessary computations on them (intersection, union, membership). The procedure gdev then splits the computation and attempt to maintain the number of cases as small as possible. This is how the odd numbers remain grouped in the previous example.

With this first section, we have given a general view of the capabilities of our library in the case of expressions in closed form. This in turn will become the basic engine for specific applications such as the ones we describe in the next two sections.

\section{Expansions of coefficients of generating functions}

By definition, the generating function of a sequence $f_{n}$ is

$$
f(z)=\sum f_{n} z^{n}
$$

When the generating function assumes a closed form, it is generally possible to retrieve the asymptotic behaviour of its coefficients. A method of singularity analysis was initiated by Darboux [3] and extended to logarithmic cases by Jungen [11] and Flajolet and Odlyzko [6]. When this method does not apply, one resorts to saddle point methods such as described by Hayman [10] and others [9, 12, 18]. These strategies are implemented in our procedure equivalent which is part of the larger system $\Lambda_{\Upsilon} \Omega$, designed to perform an automatic analysis of algorithms and data structures (see [7]).

The synopsis of this function is

where

equivalent (expr, $\operatorname{var} 1, \operatorname{var} 2,<$ nbterms $>$ )

- expr is the generating function to be studied,

- var1 is the main variable in expr,

- var2 is the index variable which will be used in the result,

- nbterms is an optional integer specifying the number of desired terms (default is 1). 


\section{II.1 Algebraic singularities}

Basically, the principle of the method is to notice that the coefficients of an analytic function with algebraic singularities are asymptotically determined by its singularities and its local behaviour in their neighbourhood. For instance $^{\dagger}$

$$
\left[z^{n}\right]\left[\frac{3}{(1-z)^{2}}+\frac{5}{\sqrt{3+z}}\right]=3(n+1)+\frac{5}{(-3)^{n} \sqrt{\pi n}}+O\left(\frac{1}{3^{n} n^{3 / 2}}\right)
$$

illustrates the general rule: the singularities are $\rho_{1}=1$ and $\rho_{2}=-3$. The local expansion about $\rho_{1}$ is $3 /\left(1-z / \rho_{1}\right)^{2}$, hence a contribution of $3(n+1) \rho_{1}^{(-n)}$ to the coefficient. Similarly the local expansion about $\rho_{2}$ is $5 / \sqrt{1-z / \rho_{2}}$, hence a contribution of $5 \rho_{2}^{-n} / \sqrt{\pi n}$. Thus the general algorithm for analytic functions with algebraic singularities is the following:

1. Find the singularities.

2. Find the local expansions.

3. Translate these expansions into expansions of the coefficients.

4. Sum these contributions.

A further optimization is achieved by considering only those singularities of smallest modulus, since the other ones will give exponentially smaller contributions to the coefficients.

EXAMPLE 12: Bernoulli numbers which are defined by their generating function, namely

$$
\frac{z}{e^{z}-1}=\sum_{n=0}^{\infty} \frac{B_{n}}{n !} z^{n}
$$

They appear in many classical formulae such as the Taylor series of several trigonometric functions, the Euler-MacLaurin formula for summations, Stirling's formula...

Since their generating function has two equimodular smallest singularities $(2 i \pi,-2 i \pi)$, we obtain an oscillating result:

$>$ equivalent $(z /(\exp (z)-1), z, n, 5)$;

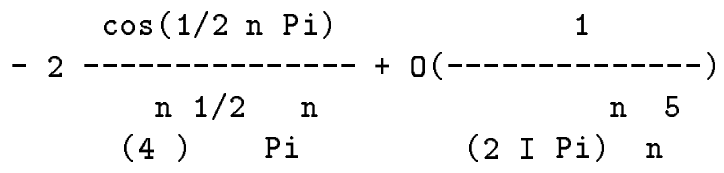

\section{II.2 Logarithmic singularities}

When the singularities are not algebraic, some transfer theorems are still available. These theorems [11,6] enable us to use the same algorithm as before with a slight modification in step 3, when we translate the local expansion into the coefficients' expansion.

Example 13: Children rounds are defined [16] as sets of cycles with a child in the middle of each cycle. The number of children rounds with $n$ children gives rise to a nice expansion (gamma is Euler's $\gamma$ constant, $\gamma \approx 0.5772156)$ :

$>$ equivalent $\left((1-z)^{\wedge}(-z), z, n, 5\right)$;

\footnotetext{
${ }^{\dagger}$ We recall the classical notation $\left[z^{n}\right] f(z)$ meaning "the coefficient of $z^{n}$ in the Laurent expansion of $f$ about 0 ".
} 


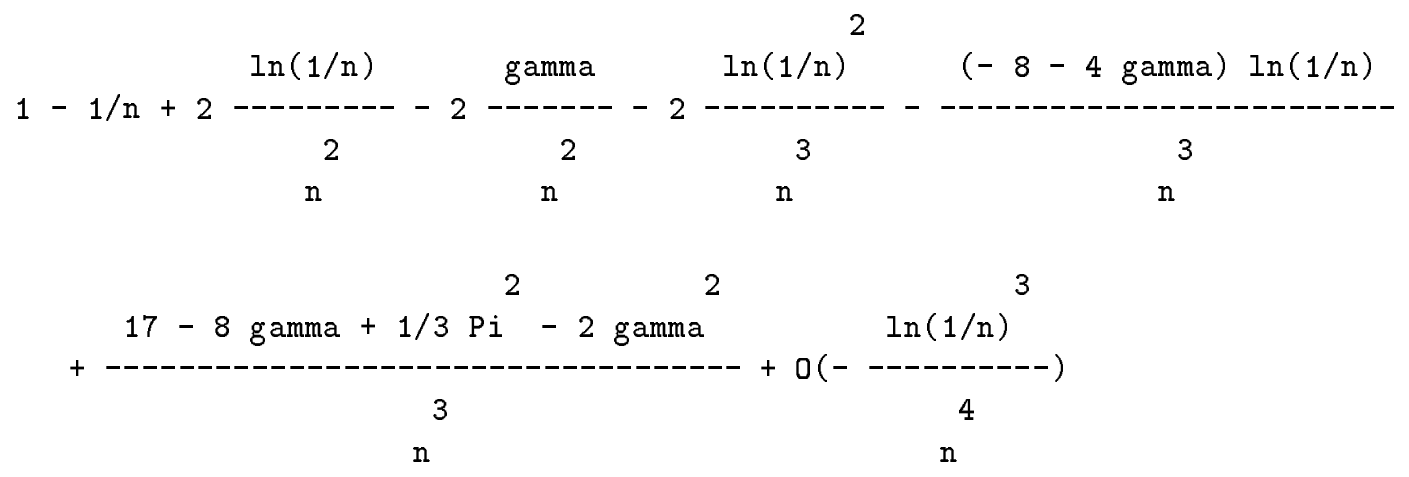

\section{II.3 Entire functions}

When the generating function is entire or has essential singularities, it is no longer possible to find the asymptotic expansion of its coefficients by singularity analysis; instead one uses saddle point methods [10, 9, $12,18]$ which find a suitable path of integration around the origin, through this special point called saddle point and then apply Cauchy's formula. The next example is an application of this method.

Example 14: Bell numbers enumerate the number of partitions of a set into non-empty subsets. Their generating function is

$$
B(z)=e^{e^{z}-1} .
$$

The asymptotic of Bell numbers is a quite difficult problem to which are devoted several pages in the book by de Bruijn [4], here the expansion is derived automatically:

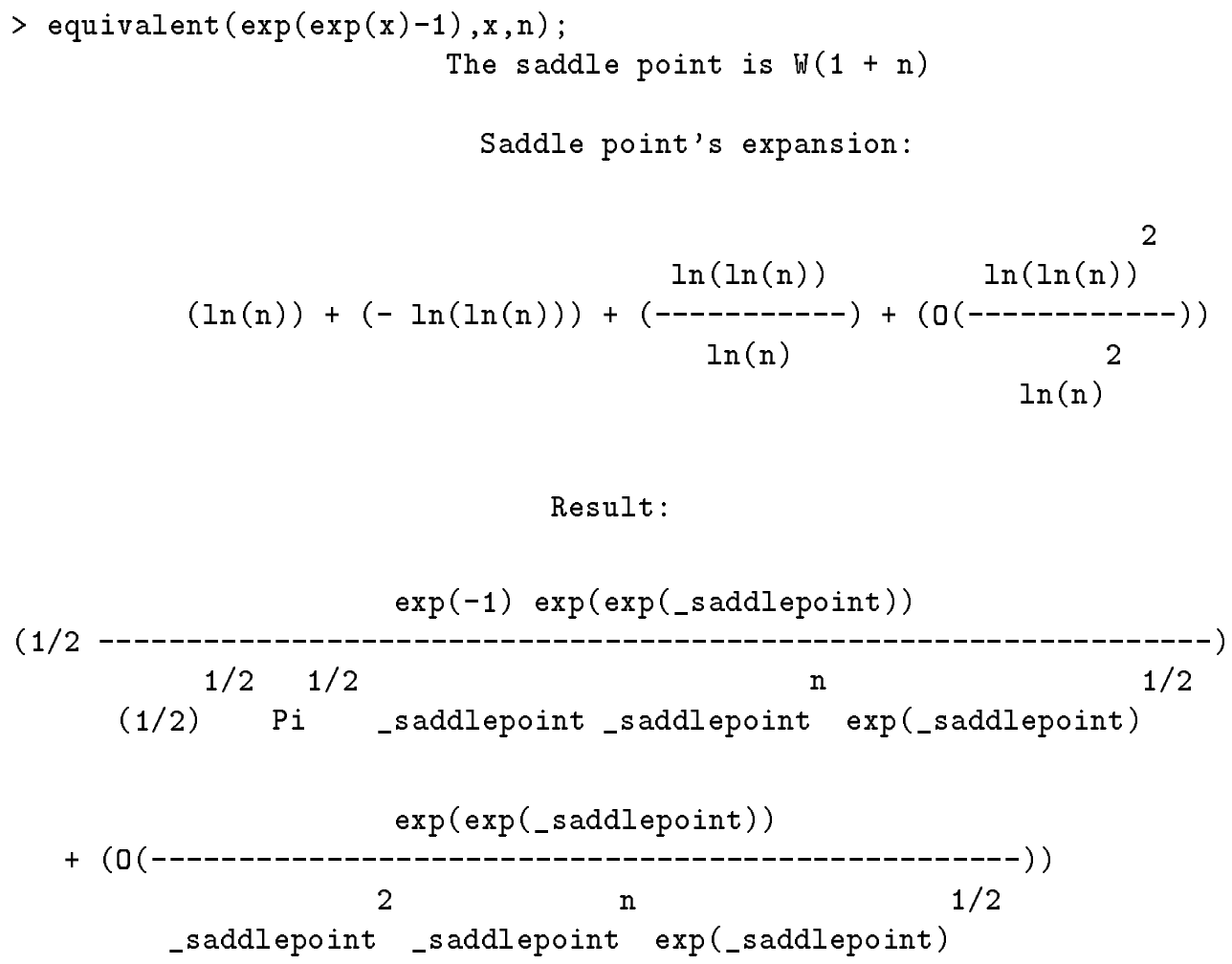


In this case, the saddle point's expansion cannot be substituted in the final result, since the scales are incompatible (one cannot get a convergent expansion). This is always the case when the generating function grows fast enough.

\section{II.4 Singularities in implicit form}

It may happen that one cannot find an explicit form for the singularities (e.g. when they are roots of a high degree polynomial). Then the computation is carried out with as much information as we can obtain about the singularities. This of course makes the decision problem for constants more difficult, but not always impossible:

EXAMPLE 15:

$>$ equivalent $(1 /(1-z * \ln (1 /(1-z))), z, n, 5)$;

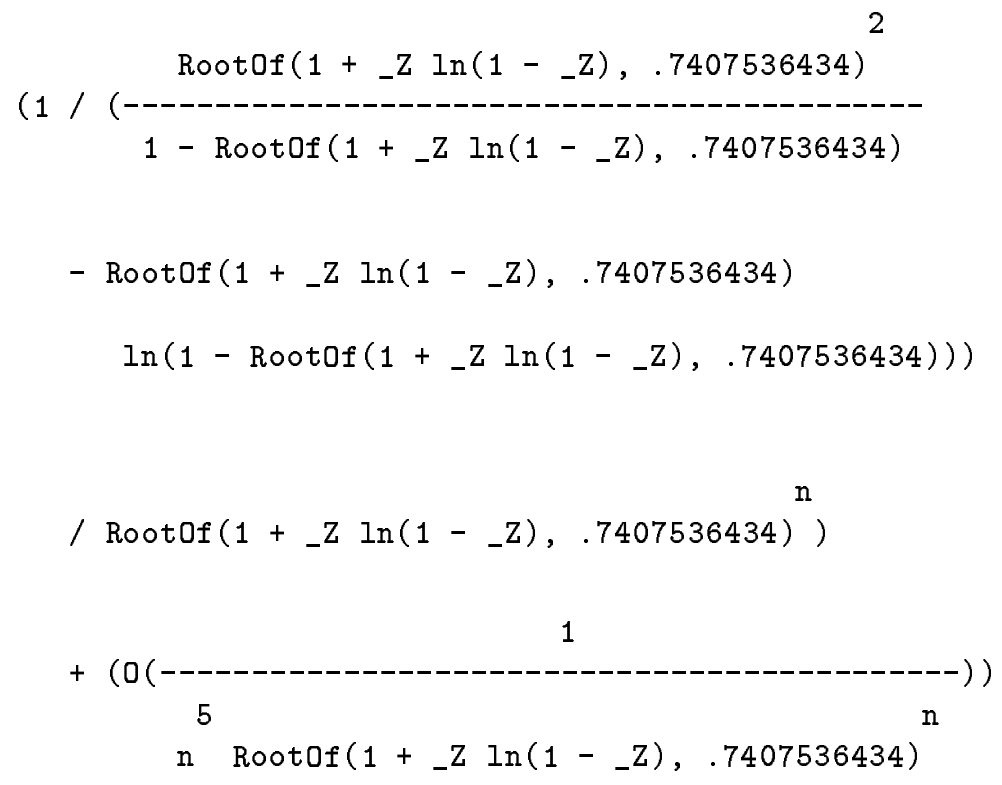

where $\operatorname{RootOf}(1+z \ln (1-z), \alpha)$ means the root of

$$
1+z \ln (1-z)=0
$$

which is the closest to $\alpha$.

\section{Asymptotic expansions of integrals}

Still based on the engine detailed in the first section - the asymptotic expansion of expressions given in closed form-we present here an application to the asymptotic expansions of integrals of the type

$$
\int_{a(z)}^{b(z)} F(z, t) d t
$$

when $z$ tends to a real limiting point (a real constant or plus or minus infinity). Of course we cannot handle so wide a class of integrals, but a large subclass can receive an automatic treatment. 
The principle of this method is to use Parseval formula for Mellin transforms (see e.g. [2]). One starts by trying to find a change of variables which transforms the integral into the nicer form

$$
\int_{0}^{\infty} f(z t) g(t) d t
$$

this first step being carried out by pattern-matching together with a possible computation of functional inverse. Then, under certain conditions, Parseval formula gives the following identity

$$
\int_{0}^{\infty} f(z t) g(t) d t=\frac{1}{2 i \pi} \int_{c-i \infty}^{c+i \infty} z^{-t} M[f ; t] M[g ; 1-t] d t
$$

where $M[f ; z]$ denotes the Mellin transform of $f$ defined by

$$
M[f ; z]=\int_{0}^{\infty} f(t) t^{z-1} d t,
$$

and $c$ lies in the common strip of analyticity of $M[f ; t]$ and $M[g ; 1-t]$. Mellin transforms can be computed in the same manner as Laplace transforms using an extensive table together with a pattern-matching which takes into account properties of the transform. The next step consists in shifting the path of integration to the right, thus expanding the integral into a sum of residues and a remaining integral. Under suitable conditions, this last integral can be bounded and one obtains a true asymptotic expansion,

$$
\int_{0}^{\infty} f(z t) g(t) d t=-\sum_{c<\Re \alpha<C} \operatorname{Res}\left(z^{-t} M[f ; t] M[g ; 1-t] ; t=\alpha\right)+O\left(z^{-C}\right)
$$

where the sum is taken over all the singularities of the integrand whose real part is greater than $c$ and smaller than $C$.

The automation of this process is (almost) straightforward when one of the following cases occurs:

$$
f(x) \approx e^{-d x^{r}} \sum_{i=0}^{\infty} \sum_{j=0}^{m_{i}} c_{i j} \ln (x)^{j} x^{-\alpha_{i}}
$$

at infinity, with $\Re(d)>0$ or $d$ imaginary, $c_{i j}$ complex numbers, $m_{i}$ finite integers and $\alpha_{i}$ complex numbers with increasing real parts, or

(ii)

$$
g(x) \approx e^{-c / x^{k}} \sum_{i=0}^{\infty} \sum_{j=0}^{n_{i}} a_{i j} \ln (x)^{j} x^{\beta_{i}}
$$

about 0 , with $\Re(c)>0$ or $c$ imaginary, $a_{i j}$ complex numbers, $n_{i}$ finite integers and $\beta_{i}$ complex numbers with increasing real parts.

In fact in both cases, the singularities of the Mellin transforms, as well as the singular part of their local expansions about these singularities can be deduced directly from the above formulae, without computing explicitly the Mellin transform (which might not be possible).

In case (i), when $d=0$ the singularities of $M[f ; z]$ are at the $\alpha_{i}$ and the singular part of its local expansion can be deduced from $d$ and the $c_{i j}$. When $d \neq 0$, then the singularities of $M[f ; t]$ lie in the left half-plane and we do not need to worry about them.

Similarly in case (ii), when $c=0$ the singularities of $M[g ; 1-z]$ are at the $1+\beta_{i}$ and the singular part of its local expansion about the singularities can be deduced from $c$ and the $a_{i j}$. When $c \neq 0$, the singularities do not bother us. 
Of course these two cases may happen together, which makes the job harder if some singularities collide, since then the knowledge of both the expansions is not enough to compute the residue of the product about a common singularity.

To sum up, the algorithm is the following:

1. Compute the expansion of $f$ at infinity and check that it fits in a scale such as the one used in (3).

2. Compute the expansion of $g$ about 0 and check that it fits in a scale such as the one used in (4).

3 . If $d \neq 0$ and $c \neq 0$, then the expansion of (1) is exponentially decreasing and we cannot find it without a change of variable (not automated).

4. From the expansions check that the strips of absolute convergence of the transforms overlap, otherwise give up.

5. If $d \neq 0$ and $c=0$, then compute explicitly $M[f ; z]$ and translate the expansion (4) into (2).

6. If $c \neq 0$ and $d=0$, then compute explicitly $M[g ; 1-z]$ and translate the expansion (3) into (2).

7. If $c=0$ and $d=0$, then compute both $M[f ; z]$ and $M[g ; 1-z]$ and still use the information given by (3) and (4) to compute the residues at non colliding singularities, part of the residues otherwise.

It is important to notice that this algorithm relies on being able to expand an expression in rather general scales and cannot be implemented without the tools described in the first section. There follows a few examples of the use of this part of our library.

\section{III.1 The $E_{1}$ function}

The exponential integral $E_{1}$ is defined by

$$
E_{1}(z)=\int_{1}^{\infty} \frac{e^{-z u}}{u} d u \quad(|\arg z|<\pi)
$$

from which an asymptotic expansion is easily computed:

EXAMPLE 16:

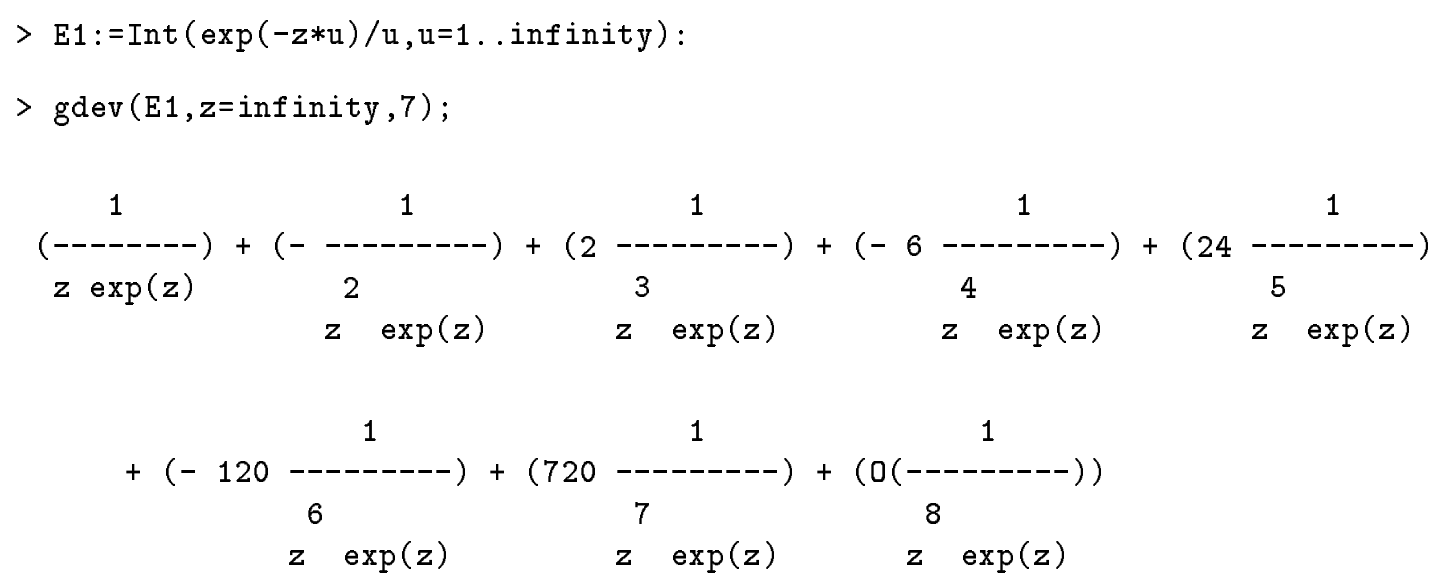

Which yields automatically the first terms of the classical expansion:

$$
E_{1}(z) \approx e^{-z} \sum_{n \geqslant 0} \frac{(-1)^{n} n !}{z^{n+1}} .
$$




\section{III.2 The Stieltjes transform of an oscillating term}

We recall that the Stieltjes transform of a function $f$ is defined by

$$
\mathcal{S}[f ; x]=\int_{0}^{\infty} \frac{f(\tau)}{x+\tau} d \tau
$$

(see e.g. [17]).

EXAMPLe 17: This is the asymptotic expansion of $\mathcal{S}[\sin (1 / t) / t ; x]$ when $x$ tends to infinity:

$>S:=\operatorname{Int}(\sin (1 / t) / t /(t+x), t=0$. infinity $)$;

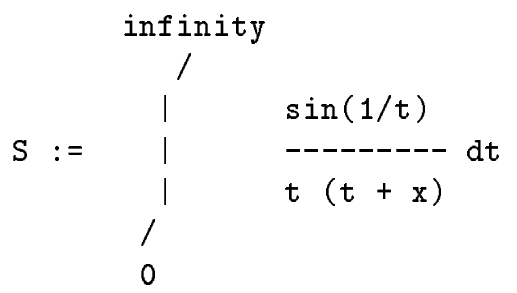

In this case, the program finds the change of variable $t=1 / u$ and using the previous notations we have $f(z)=1 /(1+z)$ and $g(z)=\sin (z)$, hence $d=0, c=0$ and the singularities of $M[g ; 1-z]$ are the positive even integers, where they collide with half of the singularities of $M[f ; z]$ since these lie at all the positive integers. The program will compute the Mellin transforms

$$
M[f ; s]=\Gamma(s) \Gamma(1-s), \quad M[g ; 1-s]=\cos \left(\frac{\pi s}{2}\right) \Gamma(1-s),
$$

and then will compute the residues. Note that in this collision of singularities logarithmic terms will appear:

$>\operatorname{gdev}(\mathrm{S}, \mathrm{x}=$ infinity, 4$)$;

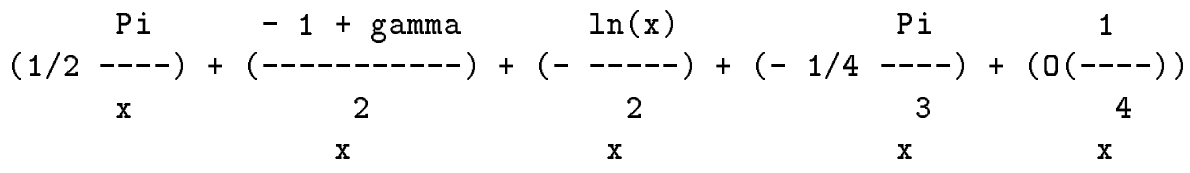

\section{III.3 Stieltjes transform with a logarithmic term}

EXAMPLE 18:

$>\mathrm{S}:=\operatorname{Int}(\operatorname{sqrt}(\mathrm{t}) * \exp (-\mathrm{t}) * \log (\mathrm{t}) /(1+\mathrm{t} * \mathrm{x}), \mathrm{t}=0$. infinity $)$

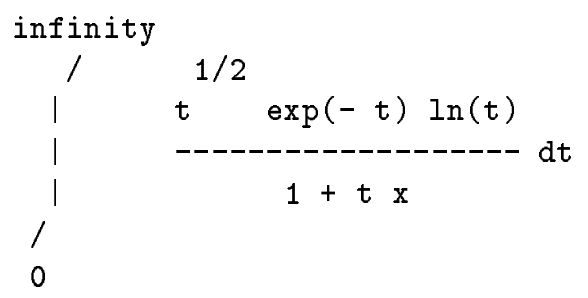

In this example (with the same notations), $f$ is the same as above, but $g$ has an algebraico-logarithmic singularity at the origin. The logarithm will induce a logarithm in the expansion and similarly the square root will produce half-integer exponents: 
$>\operatorname{gdev}(\mathrm{S}, \mathrm{x}=\inf$ inity, 3$)$;
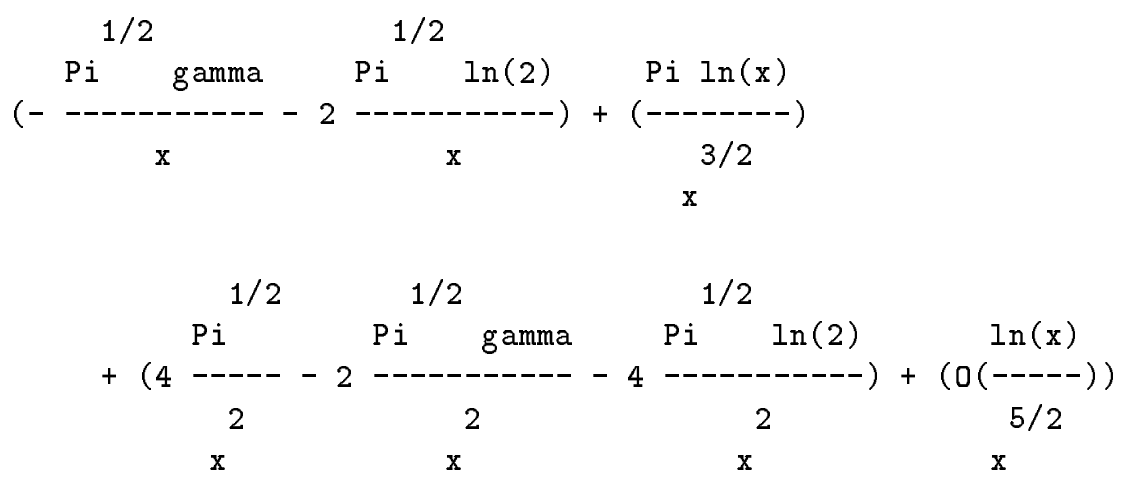

\section{III.4 Generalized Stieltjes transform}

The generalized Stieltjes transform of a function $f$ is defined by

$$
\mathcal{S}_{\mathcal{G}}[f ; x, u]=\int_{0}^{\infty} \frac{f(\tau)}{(x+\tau)^{u}} d \tau
$$

(see [17]). Once again, this leads to automatic expansions:

EXAMPLE 19:

$>S:=\operatorname{Int}\left(\exp \left(-1 / t^{\wedge} 2\right) / t /(t+x)^{\wedge}(1 / 2), t=0\right.$. infinity $)$

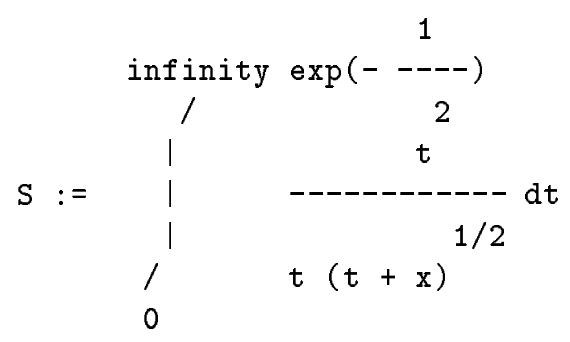

As above, the program finds the change of variable $t=1 / u$, then $f=1 / \sqrt{1+x}$ is algebraic at infinity and its transform has singularities at the half-integers, where they collide with half the singularities of the transform of $g=\exp \left(-u^{2}\right) / \sqrt{u}$, whence the logarithms and the non-integer exponents in the result:

$>\operatorname{gdev}(\mathrm{S}, \mathrm{x}=$ infinity, 4$)$;

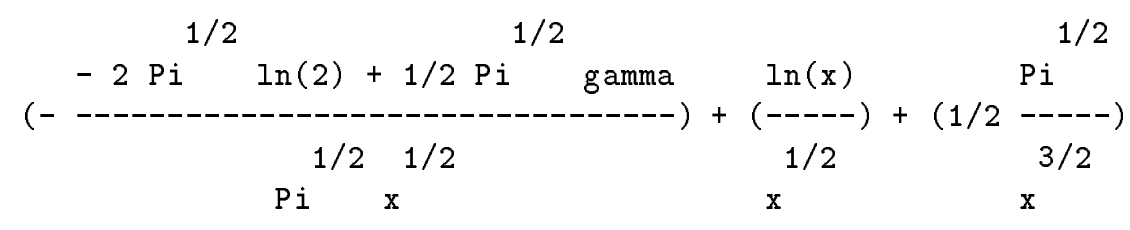




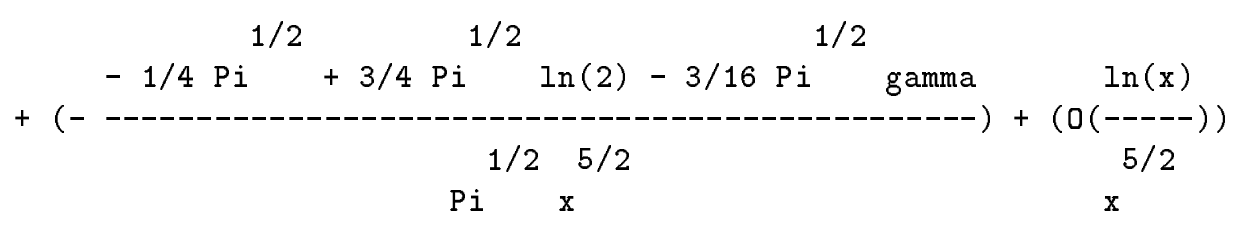

Note the difference between these last two examples. In the first one there was no collision of singularities: the terms with integer exponents came from the singularities induced by the expansion of $f$ at infinity and the logarithms together with the half-integer exponents came from the expansion of $g$ about the origin. In the second example, there was no logarithm in any local expansion, but $f$ and $g$ gave rise to singularities at the half-integers, and from this conjunction the logarithms were born.

\section{III.5 An example with the Airy function}

The Airy function is defined by

$$
\operatorname{Ai}(x)=\frac{1}{\pi} \int_{0}^{\infty} \cos \left(\frac{1}{3} t^{3}+x t\right) d t
$$

(see for instance [1]) and satisfies the remarkably simple differential equation

$$
w^{\prime \prime}-z w=0 .
$$

Once the procedure of our first section knows how this function behaves at 0 , it is not difficult to obtain non trivial expansions:

EXAMPLE 20:

$>\mathrm{S}:=\operatorname{Int}\left(\mathrm{Ai}(\mathrm{t} * \mathrm{x}) * \mathrm{t}^{\wedge}(-1 / 2) * \sin \left(\mathrm{t}^{\wedge}(3 / 2)\right), \mathrm{t}=0\right.$. . infinity $)$

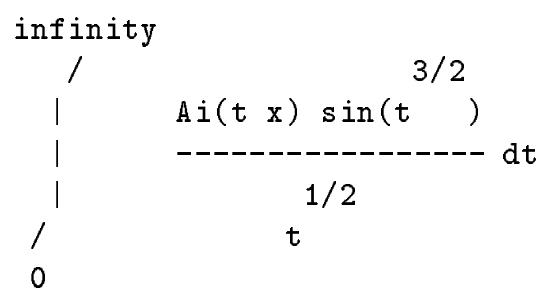

Still using the same notations, $f(z)=\operatorname{Ai}(z)$ is exponentially decreasing at infinity, while $g(z)=\sin \left(z^{3 / 2}\right) / \sqrt{z}$ is algebraic at the origin with an expansion inducing singularities at the integers $3 k+2$, the computation of the residues comes down to finding a closed form to $M[\mathrm{Ai} ; s]$. Inputing this into the program gives:

$$
M[\mathrm{Ai} ; s]=\frac{3^{\frac{4 s-7}{6}} \Gamma\left(\frac{s}{3}\right) \Gamma\left(\frac{s+1}{3}\right)}{2 \pi} .
$$

$>\operatorname{gdev}(\mathrm{S}, \mathrm{x}=$ infinity, 5$)$;

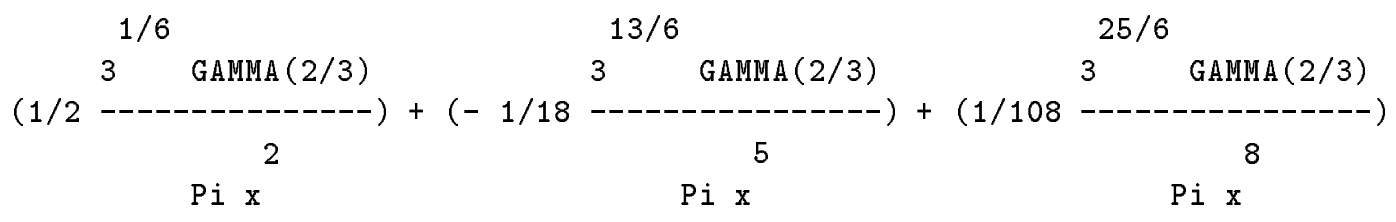




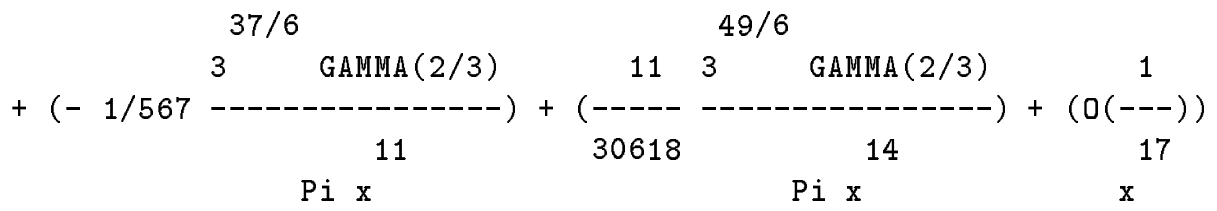

\section{Conclusion}

Non trivial automatic asymptotic expansions can be derived, provided asymptotic scales are carefully handled. Once a good engine for performing expansions in large scales is implemented, expansions which were not even expressible in symbolic computation systems previously can be obtained quite easily. Work is in progress to take benefit of this implementation of asymptotic scales to program various asymptotic methods in a general setting. The nuts and bolts of our implementation of asymptotic scales will be described elsewhere (see [14] for a preliminary report).

\section{References}

[1] Abramowitz, M., And Stegun, I. A. Handbook of Mathematical Functions. Dover, 1973.

[2] Bleistein, N., and Handelsman, R. A. Asymptotic Expansions of Integrals, 2nd ed. Holt, Rinehart and Winston, New York, 1975. Reprinted by Dover, 1986.

[3] Darboux, G. Mémoire sur l'approximation des fonctions de très grands nombres, et sur une classe étendue de développements en série. Journal de Mathématiques Pures et Appliquées (Février 1878), $5-56,377-416$.

[4] De Bruijn, N. G. Asymptotic Methods in Analysis, third ed. North Holland, 1958. Reprinted by Dover, 1981.

[5] Erdélyi, A., And Wyman, M. The asymptotic evaluation of certain integrals. Archive for Rational Mechanics and Analysis 14 (1963), 217-260.

[6] Flajolet, P., And Odlyzko, A. M. Singularity analysis of generating functions. SIAM Journal on Discrete Mathematics 3, 1 (February 1990). To appear. (Also available as INRIA Research Report 826, 1987, 25 pages).

[7] Flajolet, P., Salvy, B., and Zimmermann, P. Lambda-Upsilon-Omega: An assistant algorithms analyzer. In Applied Algebra, Algebraic Algorithms and Error-Correcting Codes (1989), T. Mora, Ed., vol. 357 of Lecture Notes in Computer Science, pp. 201-212. (Proceedings AAECC'6, Rome, July 1988).

[8] Hardy, G. H. Orders of infinity. Cambridge Tracts in Mathematics 12 (1910).

[9] Harris, B., And Schoenfeld, L. Asymptotic expansions for the coefficients of analytic functions. Illinois Journal of Mathematics 12 (1968), 264-277.

[10] Hayman, W. K. A generalization of Stirling's formula. Journal für die reine und angewandte Mathematik 196 (1956), 67-95.

[11] Jungen, R. Sur les séries de Taylor n'ayant que des singularités algébrico-logarithmiques sur leur cercle de convergence. Commentarii Mathematici Helvetici 3 (1931), 266-306.

[12] Odlyzko, A. M., And Richmond, L. B. Asymptotic expansions for the coefficients of analytic generating functions. Aequationes Mathematicae 28 (1985), 50-63. 
[13] Richardson, D. Some undecidable problems involving elementary functions of a real variable. The Journal of Symbolic Logic (1968), 514-520.

[14] Salvy, B. Fonctions génératrices et asymptotique automatique. Research Report 967, Institut National de Recherche en Informatique et en Automatique, 1989.

[15] Schmidt, H. Beiträge zu einer Theorie der allgemeinen asymptotischen Darstellungen. Mathematische Annalen 113 (1936), 629-656.

[16] Stanley, R. P. Generating functions. In Studies in Combinatorics, M.A.A. Studies in Mathematics, Vol. 17. (1978), G.-C. Rota, Ed., The Mathematical Association of America, pp. 100-141.

[17] Widder, D. V. The Laplace Transform. Princeton University Press, 1941.

[18] Wyman, M. The asymptotic behavior of the Laurent coefficients. Canadian Journal of Mathematics 11 (1959), 534-555. 